Ann. Biol. anim. Bioch. Biophys., I974, 14 (4-A), 623-632.

\title{
ÉTUDE DU NOMBRE D'OVULATIONS APRES SUPEROVULATION INDUITE PAR PMSG CHEZ TROIS SOUGHES DE RATTES ADULTES
}

\author{
J.-C. MARIANA \\ avec la collaboration technique de M. J. MANICOM \\ Station de Physiologie de la Reproduction, \\ Centre de Recherches de Tours, I. N.R. A., \\ B.P. 1, Nouzilly, 37380 Monnaie
}

\section{RÉSUMÉ}

Trois souches de rattes sélectionnées pour leur sensibilité à FSH (test de STEELMAN et Pohley) manifestent une sensibilité à PMSG différente quand elles sont adultes (nombre d'ovulations).

La courbe de réponse des 3 souches présente un maximum pour des doses voisines de PMSG ; les animaux n'ovulent pas au-delà de I6o UI.

La dispersion des réponses présente également un maximum correspondant à la même dose de PMSG, soit 3o UI. Jusqu'à 30 UI de PMSG la réponse est vraisemblablement fonction de la sensibilité ovarienne. Au-delà il y a lutéinisation de certains follicules et le nombre d'ovulations décroît.

L'étude de l'influence de PMSG sur le nombre des ovulations montre que PMSG introduit une source de variation qui n'existe pas lors de l'ovulation naturelle. Ainsi le coefficient de variation du nombre des ovulations de plusieurs souches de rat décroît quand le nombre moyen des ovulations naturelles augmente, alors que dans le cas de la superovulation induite, ce même coefficient croît légèrement avec la moyenne de l'ensemble des réponses et le rapport de la variance à la moyenne du nombre d'ovulations croît très nettement avec la moyenne (FAI,CONER et al., I96I).

Beaucoup d'auteurs ont remarqué que la variabilité des réponses augmentait en même temps que la dose de PMSG injectée (GREENwALD, I972 : Hamster ; MARIANA et al., I970 : Vache; WELSCHEN et RUTTE, I972 : Rat), et ceci chez les immatures comme chez les adultes (YING et MEYER, I973; McCormaCk et MEYER, r963 : Rat).

Nous avons done essayé d'analyser sur trois souches de rattes, la part de la variation liée au génotype et celle liée au traitement par PMSG. L'argument cité par LAND et FALCONER (I969) selon lequel une souche peut pondre plus d'œufs 
qu'une autre pour une faible dose de PMSG et moins pour une dose supérieure ayant retenu notre attention, nous avons utilisé une large gamme de doses de PMSG sur 3 couches différentes de rattes, comme l'ont fait HuSAIN et SAUCIER (I970) sur une seule souche de rattes.

\section{MATÉRIEL, ET MÉTHODE}

Les rattes âgées de 3 mois appartiennent aux 3 souches, 03, o4 et I 2 de notre laboratoire, présentant une sensibilité différente à $\mathrm{FSH}$, les deux souches $0_{3}$ et $0_{4}$ étant à cet égard plus sensibles que la souche I2. Les rattes pesaient en moyenne environ $200 \mathrm{~g}$ (tabl. I) et étaient soumises à un rythme diurne de $14 \mathrm{~h}$ de lumière et ro $\mathrm{h}$ de nuit.

La régularité des cycles a été testée par des frottis vaginaux effectués une fois par jour à $8 \mathrm{~h} 30$ et seules les rattes manifestant les séquences : métoestrus I, metoestrus II, dioestrus, prooestrus et œstrus au cours de 2 cycles successifs de 4 jours ont été conservées. Remarquons que pour la souche I2, une ratte sur deux a des cycles de 5 jours et une sur trois a des cycles irréguliers de 4 jours (tabl. I).

TABLEAU I

Caractéristiques des trois souches

\begin{tabular}{|c|c|c|c|}
\hline & 03 & 04 & 12 \\
\hline $\begin{array}{l}\text { Pourcentages des rattes ayant } \\
\text { des cycles irréguliers de } 4 \text { jours }\end{array}$ & $20(1)$ & 13,6 & 54,1 \\
\hline $\begin{array}{c}\text { Pourcentages des rattes ayant } \\
\text { des cycles de } 5 \text { jours }\end{array}$ & 4,6 & 3,8 & 38,7 \\
\hline Poids moy. des rattes des 3 souches & 207 & 204 & 200 \\
\hline$s_{m}$ écart-type de la moyenne & $s_{m}=1,69$ & $s_{m}=1,63$ & $s_{m}=1,89$ \\
\hline
\end{tabular}

(1) Ce pourcentage est calculé à partir du pourcentage de rattes ayant des cycles de 4 jours (irréguliers ou réguliers).

Nous avons tenté d'estimer la durée des phases du cycle pour les trois souches en utilisant la méthode proposée par Arrchison et Silvey (1957). Les remarques de ces auteurs s'appliquent malheureusement à notre cas, en particulier un intervalle de $24 \mathrm{~h}$ entre deux frottis est trop important et ne permet pas d'estimation précise de la durée des différentes phases. Il semble cependant que les rattes des trois souches aient des durées d'œstrus, métoestrus, dioestrus, proœstrus très voisines et que PMSG, injecté le matin où les premiers signes vaginaux d'un métoestrus sont nets, ne modifie pas la durée du cycle.

La veille de l'œestrus présumé, les rattes ont reçu HCG, ont été mises au mâle peu après et abattues le lendemain après contrôle des frottis vaginaux ; les oviductes ont été disséqués pour la récupération des œufs.

Les doses de PMSG utilisées ont été I,25-2,5-5-10-I7,4-30,2-52,6-91,6-I59,4 UI et la dose unique d'HCG que nous avons injectée ( 150 UI) est plus forte que celle indiquée par RowLANDs (I944). Elle reste cependant bien en deça de celles étudiées par Husain et SaUcier (I970) qui ont montré que la courbe de réponse à PMSG n'était pas modifiée en valeur absolue ou relative jusqu'à une dose de 5 oo UI de HCG. 


\section{RÉSULTATS}

Le nombre d'œufs pondus croît avec la dose de PMSG et atteint un maximum pour 30,2 UI pour les souches 03 et I 2 et 52,6 UI pour la souche 04 bien que, pour cette dernière, les valeurs moyennes des réponses obtenues avec 30,2 UI et 17,4 UI ne

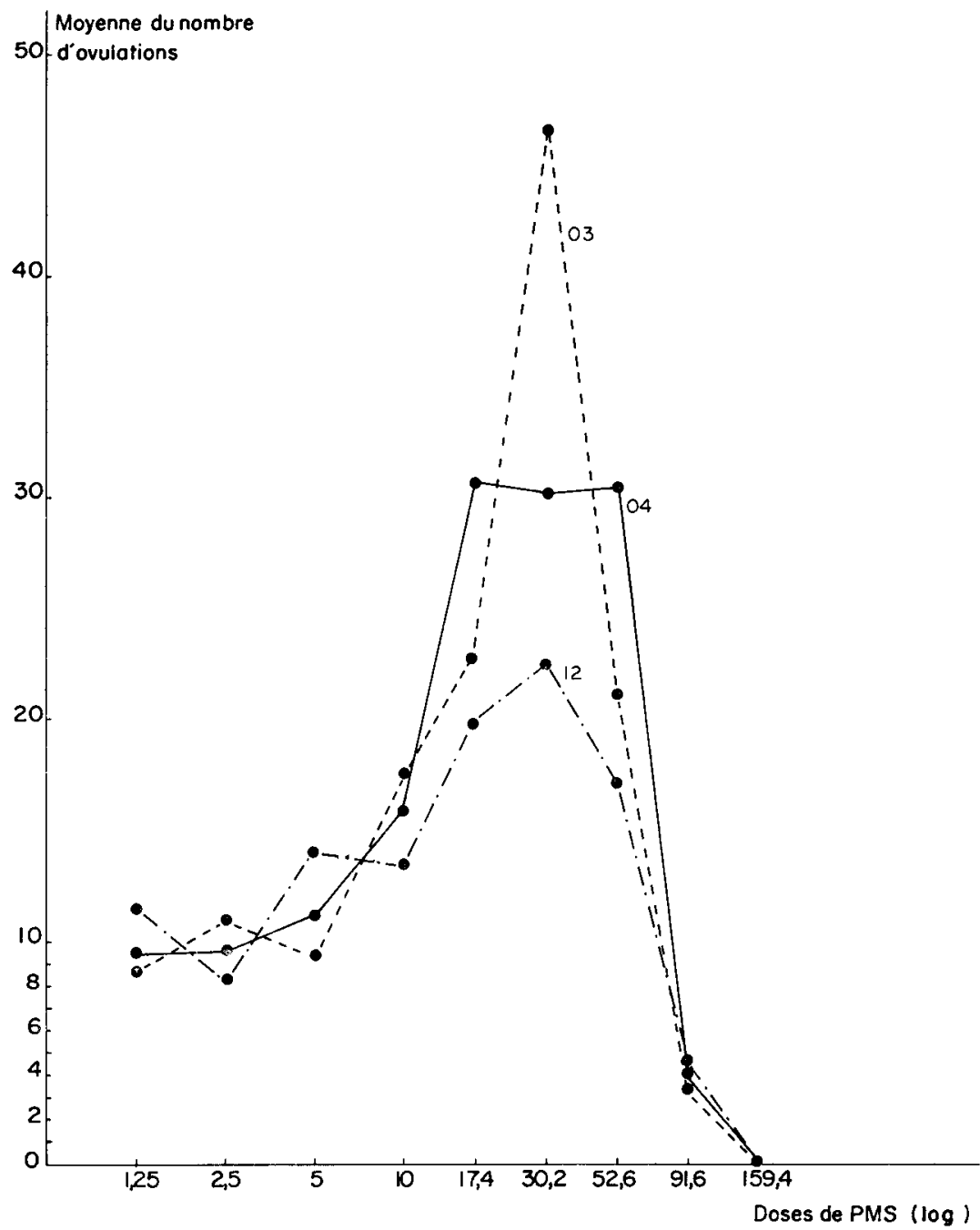

FIG. I. - Nombre moyen d'ouulations obtemues chez 3 souches de rattes adultes (03-O4-I2) injectées avec différentes doses de PMSG

soient pas significativement différentes de celles obtenues avec 52,6 UI. Pour l'ensemble des souches, le nombre d'ovulations décroît ensuite pour s'annuler, quand on traite avec I59,4 UI (tabl. 2, fig. I). 
Jusqu'à la dose de Io UI le nombre d'ovulations est voisin pour les 3 souches ; puis on observe des différences pour I 7,4 UI, 30,2 UI et 52,6 UI ; 1a souche I2 répondant toujours moins que les deux autres; à 9I,6 UI les résultats sont de nouveau voisins pour les trois souches.

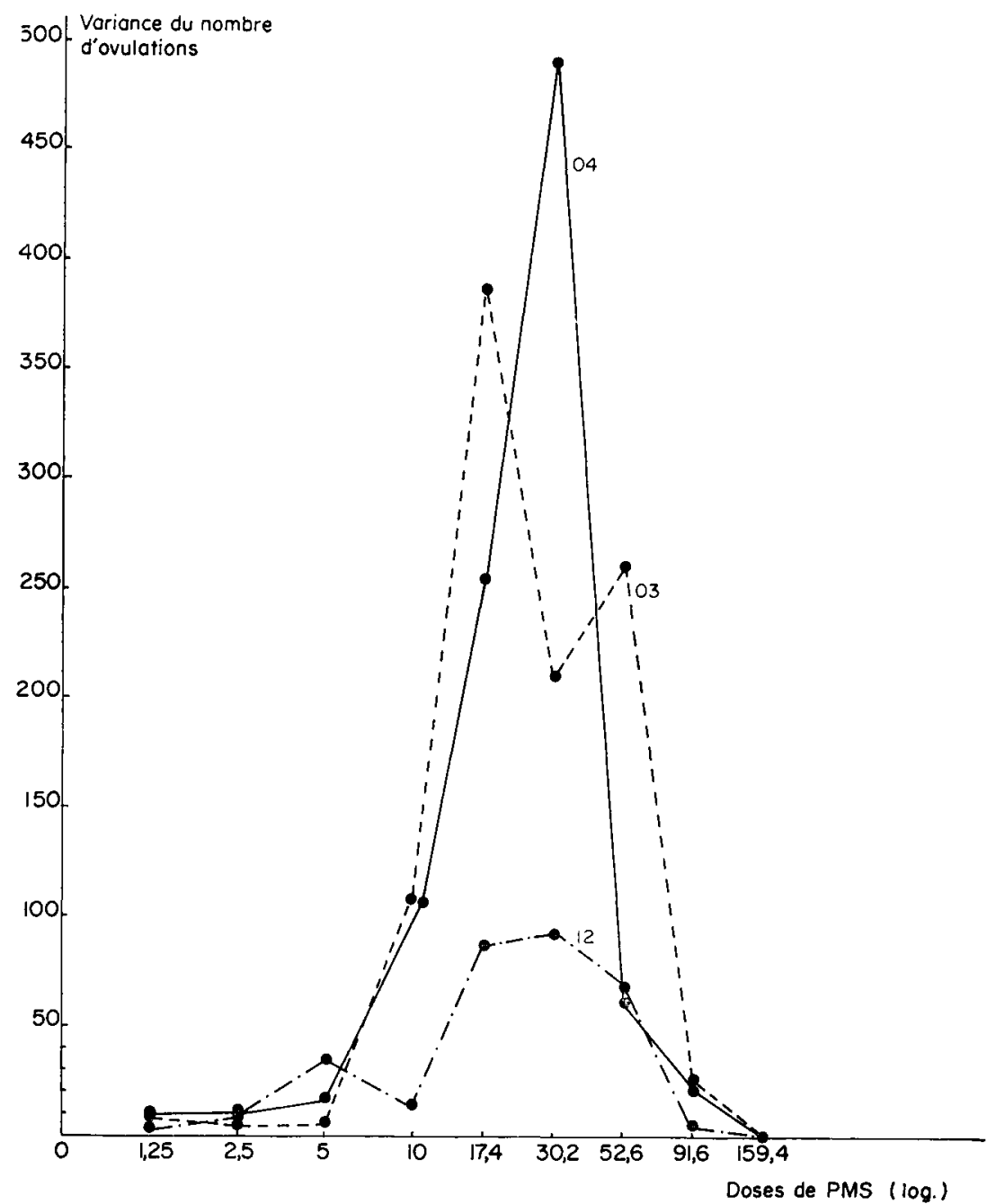

FIG. 2. - Évolution de la variance du nombre d'ovulations obtenues chez 3 souches de rattes adultes (03-04-12) injectées avec différentes doses de PMSG

La variance du nombre d'ovulations en fonction de la dose de PMSG évolue selon une courbe voisine de celle de la moyenne, les différences entre les souches étant les plus accentuées autour du maximum de réponse (fig. 2).

$\mathrm{Si}$ on classe les réponses en fonction de la moyenne du nombre d'ovulations et indépendamment de la dose (fig. 3) on constate que la variance croît généralement comme la moyenne pour les trois souches malgré quelques exceptions. 
L'examen des figures 4 et 5 montre en outre que pour une même dose d'hormone, les souches ne se classent pas de la même façon selon que l'on prend comme critère de classification le nombre moyen d'ovulations ou la variance de ce même nombre;

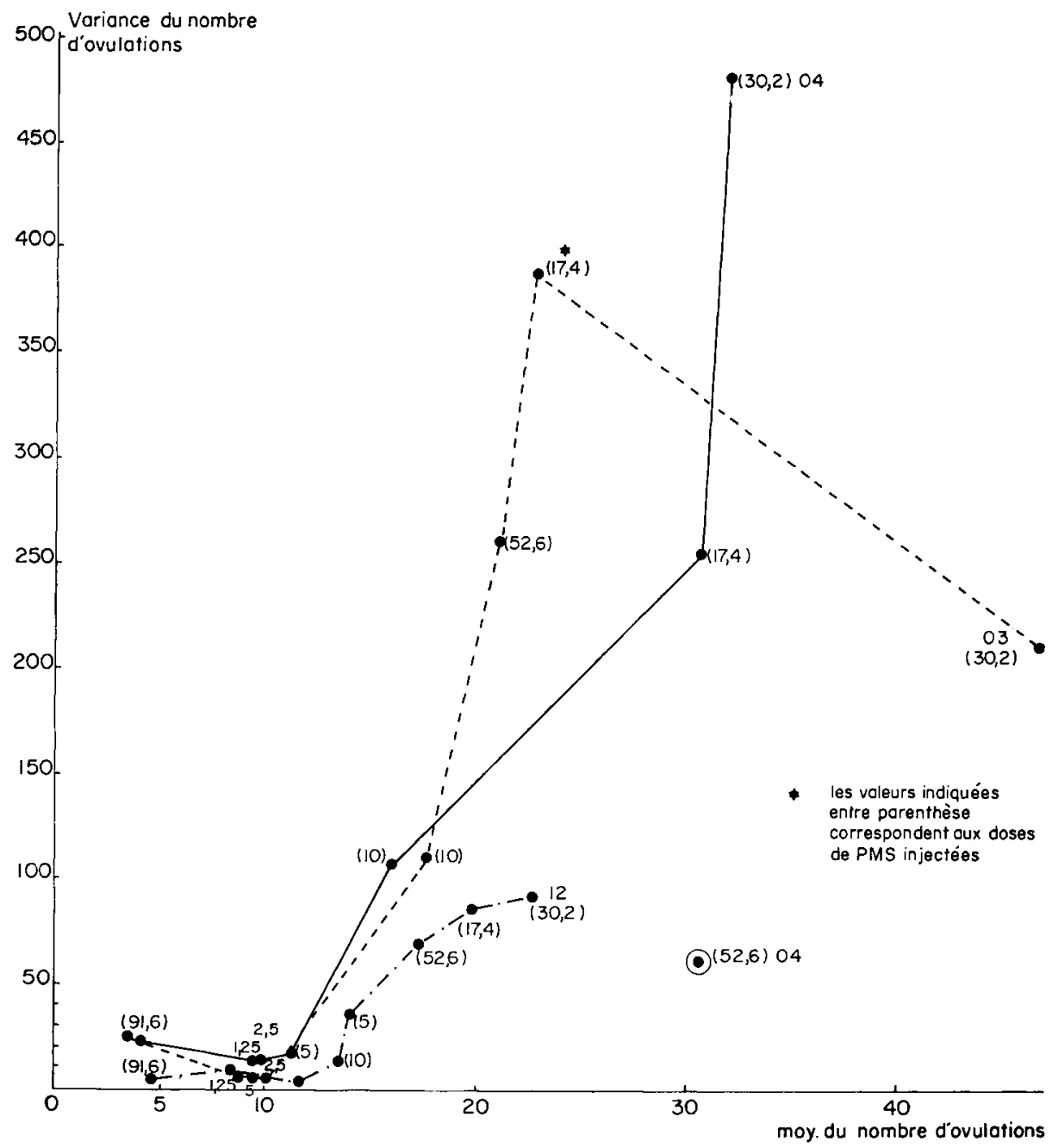

FIG. 3. - Représentation de la relation: moyenne-variance pour chaque souche et pour toute dose de PMSG. Evolution de la variance du nombre d'ovulations de 3 souches de rattes adultes $\left(\mathrm{O}_{3}-\mathrm{O}^{-12}\right)$ en fonction du nombre moyen d'ovulations $d u$ lot.

( ) : dose de PMSG injectée

ainsi la souche ayant la réponse moyenne la plus élevée n'a pas nécessairement la dispersion des réponses la plus grande au moins pour les doses I7,4 UI, 30,2 UI et 52,6 UI de PMSG. 
TABLEAU 2

Réponses des trois souches à PMS

\begin{tabular}{|c|c|c|c|c|c|c|c|c|c|c|}
\hline \multirow{2}{*}{\multicolumn{2}{|c|}{ Souche }} & \multicolumn{9}{|c|}{ PMS (UI) } \\
\hline & & 1,25 & 2,5 & 5 & 10 & 17,4 & 30,2 & 52,6 & 91,6 & 159,4 \\
\hline \multirow{4}{*}{03} & $a$ & 8,62 & 10,9 & 9,4 & 17,5 & 22,8 & 46,7 & 21,1 & 3,3 & 0 \\
\hline & $b$ & 0,89 & 0,67 & 0,76 & 3,02 & 6,22 & 4,58 & 5,10 & 1,57 & 0 \\
\hline & $c$ & 0 & 0 & 0 & 0 & 3 & 0 & 0 & 4 & 10 \\
\hline & $d$ & 10 & 10 & 10 & 10 & 10 & 10 & 10 & 10 & 10 \\
\hline \multirow{4}{*}{04} & $a$ & 9,5 & 9,6 & 11,2 & 16 & 30,7 & 32 & 35,1 & 4 & 0 \\
\hline & $b$ & 1,0 & 1 & 1,35 & 2,9 & 5 & 7 & 2,6 & 1,53 & 0 \\
\hline & $c$ & 0 & 0 & 0 & 0 & 0 & 1 & 0 & 2 & 10 \\
\hline & $d$ & 10 & 11 & 10 & 12 & 10 & 10 & 10 & 10 & 10 \\
\hline \multirow{4}{*}{12} & $a$ & 11,55 & 8,3 & 14 & 13,5 & 19,8 & 22,6 & 17,2 & 4,5 & 0 \\
\hline & $b$ & 0,64 & 1,0 & 2,2 & 1,1 & 2,9 & 4,2 & 2,6 & 0,8 & 0 \\
\hline & $c$ & 0 & 0 & 0 & 0 & 1 & 0 & 0 & 1 & 7 \\
\hline & $d$ & 9 & 8 & 7 & 10 & 9 & 7 & 10 & 9 & 7 \\
\hline
\end{tabular}

$a$ : moyenne du nombre d'ovulations

$c$ : nombre de rattes n'ovulant pas $b$ : écart type de la moyenne

$d:$ nombre de rattes total

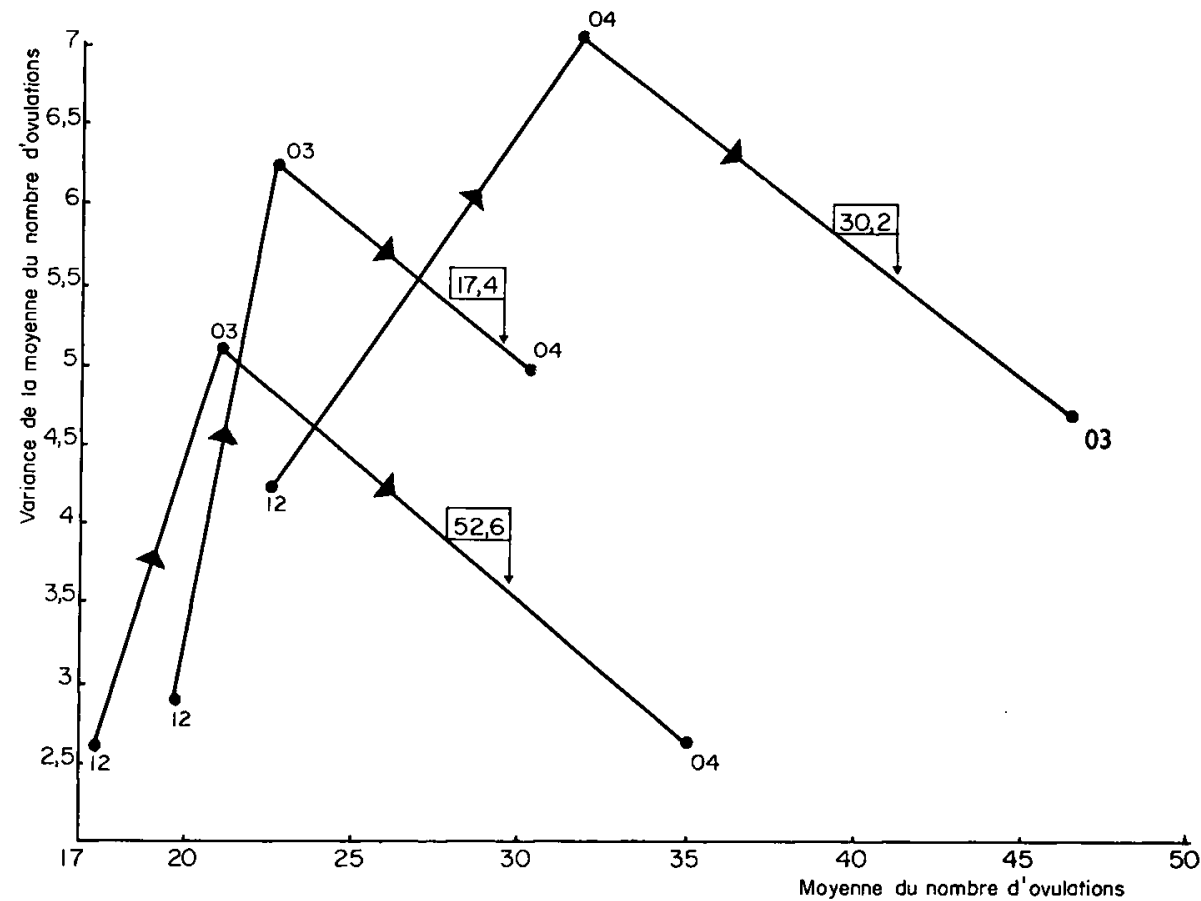

FIG. 4. - Évolution de la variance de la moyenne du nombre d'ovulations de 3 souches de rattes adultes (03-04-12) injectées de 10,4-37,2-52,6 UI de PMSG 


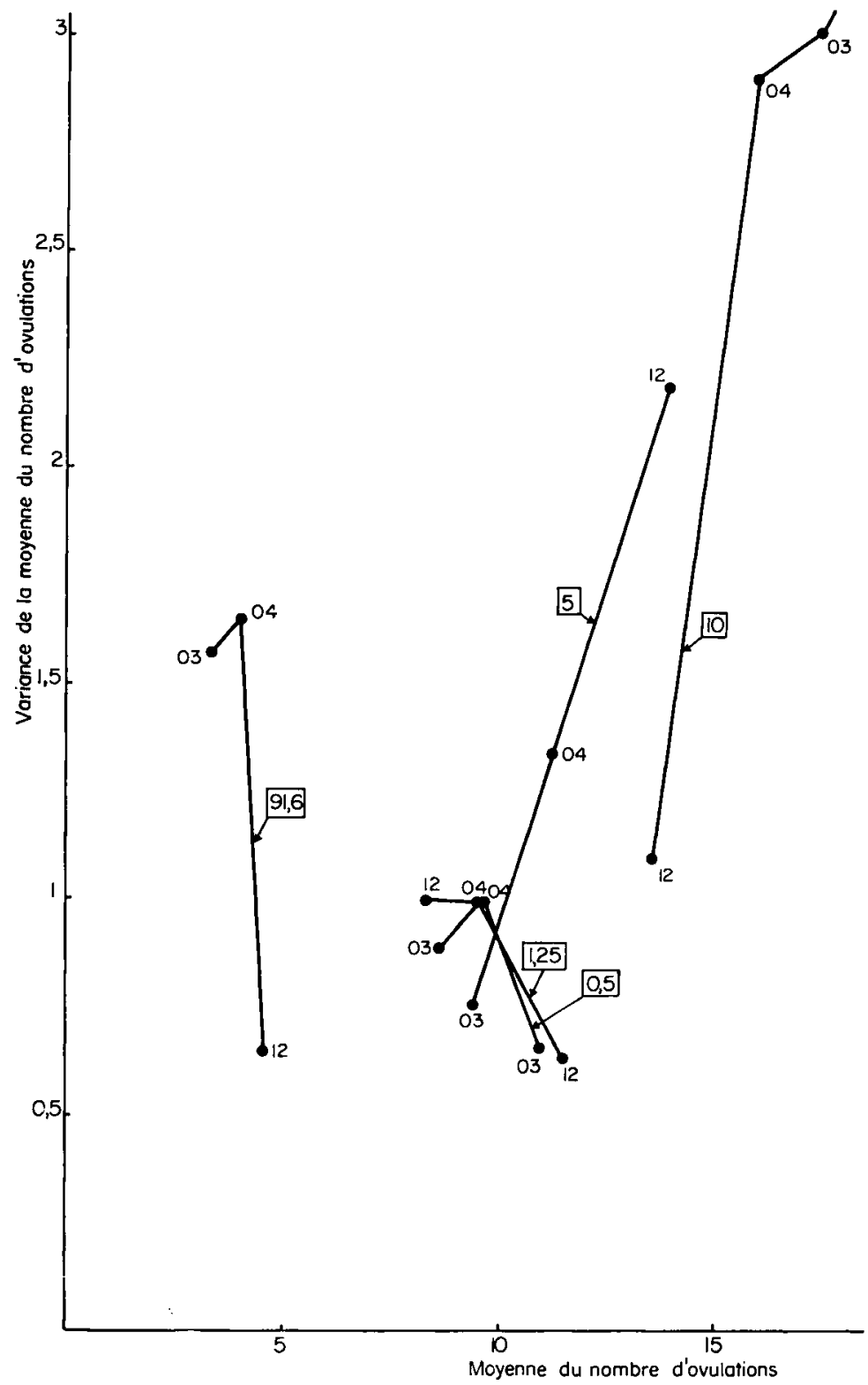

FIG. 5. - Évolution de la variance de la moyenne du nombre d'ovulations de 3 souches de rattes adultes (03-04-I2) injectées de 0,5-1,25-5-10-91,6 UI de PMSG 


\section{DISCUSSION}

I. Les rattes adultes des trois souches manifestent le même type de réponse à des doses de PMSG comprises entre I,25 UI et I59,4 UI. Jusqu'à 30,2 UI le nombre des ovulations croît, au-delà le nombre décroît.

On peut penser que jusqu'à 30,2 UI de PMSG, la stimulation directe de l'ovaire entraîne une croissance folliculaire accrue en fonction de la dose de PMSG injectée. L'analyse histologique d'ovaires de rattes traitées dans les mêmes conditions avec Io UI ou 20 UI de PMSG ont confirmé que les follicules d'une surface de section médiane supérieure à 100 ooo $\mu^{2}$ étaient d'autant plus nombreux que la dose de PMSG utilisée était plus importante. Au-delà de 30,2 UI, le nombre d'ovulations décroît ; ceci n'est vraisemblablement pas lié à une insuffisance du développement folliculaire mais à une hyperstimulation de l'ovaire dont les signes sont déjà perceptibles à 20 UI ; on peut en effet constater des débuts de lutéinisation de la granulosa de certains follicules pour cette dose de PMSG ; de tels follicules n'ovulent plus (WELSSCHEN et RuTTE, I97I).

Cette action directe de PMSG sur l'ovaire semble fonction de la sensibilité ovarienne aux hormones folliculo-stimulantes; il est vraisemblable que la courbe du nombre des ovulations en réponse à PMSG est conditionnée par l'équilibre des sécrétions hypophysaires et ovariennes.

LAND (I965) avait montré que pour les faibles doses de FSH exogène injectées à des souris adultes il y a compétition entre une activité folliculo-stimulante directe et une inhibition des sécrétions hypophysaires endogènes; la courbe du nombre des ovulations en fonction de la dose de PMSG injectée présente successivement un maximum pour les doses de PMSG de l'ordre de o,I25 UI et un minimum pour I UI, le nombre des ovulations augmente ensuite brutalement avec des doses supérieures.

YING et MEYER (I969) avaient également observé un phénomène analogue chez la ratte de 22 jours ; de faibles quantités de PMSG (6 UI) entraînent une superovulation importante, mais avec I2 UI les rattes n'ovulent pas. Elles ovulent de nouveau avec 30 UI de PMSG, il y a même alors une forte superovulation.

Chez la ratte de 30 jours, donc proche de la puberté, Sridharan et MEYER (I972) observent en réponse à PMSG une courbe d'ovulation ayant un maximum pour 55 UI de PMSG et une chute rapide entre 55 et r2o UI. C'est également le type de réponse obtenu par HuSAIN et SAUCIER (I970) et nous-même, dans l'expérience rapportée dans cet article.

2. Si les 3 souches ont le même type de courbe de réponse à PMSG la dispersion des réponses est différente. L'examen du nombre d'ovulations moyen des trois souches montre que pour chaque dose de PMSG la dispersion des réponses moyennes des trois souches croît quand on augmente la dose de PMSG et décroît pour les valeurs de PMSG supérieures à 30,2 UI.

Le motif général d'augmentation de la dispersion des réponses se retrouve donc si on considère chaque souche comme une entité. A l'intérieur d'une souche, le fait que la variance augmente avec la dose peut être dû à la variabilité génétique des animaux. On peut penser que la façon la plus valable de séparer les animaux est 
alors, soit de les sélectionner sur la sensibilité ovarienne quand ils sont immatures, soit d'utiliser chez les adultes une dose de PMSG optimale manifestant les réponses extrêmes ; ceci a été la base de l'étude de LAND et FALCONER (I969) qui, en utilisant des doses de PMSG relativement faibles (2 à $6 \mathrm{UI}$ ), sélectionnent les animaux sur le critère faible ou forte ovulation.

On peut enfin imaginer que la sensibilité ovarienne naturelle à FSH varie d'une ratte à l'autre, et que PMSG introduit une seconde source de variation. On peut alors envisager un modèle de réponse individuelle à $P M S G$, dépendant de paramètres tels que le nombre de follicules, la sensibilité à FSH etc. Pour l'ensemble de la population, les paramètres caractérisant la courbe du nombre des ovulations en fonction de PMSG auraient une distribution statistique ; on conçoit que dans un tel schéma la variance des réponses puisse augmenter en fonction de la dose.

Ce schéma est à rapprocher de celui proposé par GREENwALD pour la réponse du système folliculaire à PMSG ; il distingue en effet, parmi les follicules cycliquement utilisés, ceux qui sont sensibles à PMSG et ceux qui ne sont stimulés que pour tune dose suffisante de PMSG. Il appelle ces derniers : follicules de réserve.

De plus, si pour les doses relativement faibles de PMSG (de 5 à Io UI) on constate que la dispersion du nombre moyen des ovulations croît comme ce même nombre, il n'en est plus de même pour les doses supérieures.

FALCONER, d'ailleurs, posait cette question : "La variance augmente-t-elle avec la dose ou parce que la moyenne du nombre d'ovulations augmente elle-même?" Mais sa réponse n'était guère concluante. Nous pensons, dans le cadre de l'expérience rapportée, que l'augmentation de la variabilité des réponses est liée principalement à l'augmentation du niveau de réponse et est un problème de régulation ne dépendant que partiellement de PMSG.

$$
\text { Reçu pour publication en avril } 1974 \text {. }
$$

\title{
SUMMARY
}

\author{
STUDY OF THE NUMBER OF OVULATIONS AFTER PMSG-INDUCED
}

SUPEROVULATION IN THREE STRAINS OF ADULT RATS

Three strains of rats selected for FSH sensitivity (Steelman and Pohley's test) show a different PMSG sensitivity when they are adult (number of ovulations).

The response curve of the three strains shows a maximum for similar doses of PMSG; the animals do not ovulate over I6o IU.

The disparity of responses also has a maximum corresponding to the same PMSG dose, or $30 \mathrm{IU}$. Up to $30 \mathrm{IU}$ of PMSG, the response seems to be in terms of ovarian sensitivity. Beyond that, some follicles are luteinized and the number of ovulations decreases.

\section{RÉFÉRENCES BIBLIOGRAPHIQUES}

Aitchison J., Silvey S. D., I957. The generalization of Probit analysis to the case of multiple responses Biometrika, 44, I3I-I43.

Falconer D. S., Edwards R. G., Fowler R. E., Roberts R. C., I961. Analysis of differences in the numbers of eggs shed by the two ovaries of mice during natural cestrus or after superovulation. J. Reprod. Fert., 2, 4I 8-437. 
Greenwald G. S., 1972. Analysis of superovulation in the adult hamster. Endocrinology, 71, 378-389.

Husain S. M., SAucier R., I97o. Induction of superovulation in mature rats with gonadotrophins. Can. J. Physiol. Pharmac., 48, I96-r 99.

LAND R. B., I965. Ovarian response of mice to low doses of hormones with follicle-stimulating activity. Nature, 206, 939-940.

Land R. B., Falconer D. S., I969. Genetic studies of ovulation rate in the mouse. Genet. Res. Camb., $13,25-46$.

McCormack, Meyer R. K., 1963. Ovulation induced by progesterone in immature rats pretreated with pregnant mare serum gonadotrophin. Gen. Comp. Endocr., 3, 300-307.

Mariana J. C., Mauleon P., Benoit M., Chupin D., ig7o. Variabilité et répétabilité du nombre d'ovulations obtenues après injection de I 600 UI de PMSG et de I 500 UI de HCG. Ann. Biol. anim. Bioch. Biophys, 10, no hors série $\mathrm{I}, 47-63$.

Mariana J. C., Nguyen Huy N., 1973. Folliculogenèse chez la vache. Ann. Biol. anim. Bioch. Biophys., 13, no hors série, 2 I I-22 I.

Rowlands I. W., I944. The production of ovulation in the immature rats. J. Endocr., 3, 384-39I.

ShaO YAo YiNG, MEXER R. K., I973. Effect of progesterone on the time of ovulation in immature rats pretreated with pregnant mare's serum gonadotrophin (PMSG). Acta endocrinol., 72, 161-172.

Shao Yao Ying, MeYer R. K., I969. Dose dependant pregnant mare's serum induced ovulation in immature rats. Proc. Soc. Exp. Biol. N. Y., 130, 40-43.

SRIDharan B. N., Meyer R. K., I972. Observe of ovulatory effect of PMSG in the zo day old rat. Proc. Soc. Exp. Biol. N. Y., 140, 1493-1496.

Stenlman S. L., Pohley F. M., I953. Assay of the FSH based on the augmentation with HCG. Endocrinology, 53, 604-6I6.

Welschen R., RUtTe M., I97I. Ovulation in adult rats after treatment with pregnant mare serum gonadotrophin during ostrus. Acta endocrinol., 68, 4I-49.

Zarrow M. X., Christenson C. M., Eleftheriou B. F., ig7i. Strain differences in the ovulatory response of immature mice to PMS and to the pheromonal facilitation of PMS-induced ovulation. Biol. Reprod., 4, 52-56. 